\title{
As faces do cuidado em saúde mental na rede de atenção psicossocial: do acolhimento à desresponsabilização
}

\author{
The faces of mental health care in the psychosocial care network: from welcome to reliability \\ Las caras de la atención de salud mental en la red de atención psicosocial: de la bienvenida a la \\ fiabilidade
}

\begin{abstract}
Resumo
A produção do cuidado no campo da saúde mental floresce a partir do encontro entre profissionais e usuários. O estudo objetivou-se analisar o cuidado em saúde mental na rede de atenção psicossocial a partir da prática do acolhimento. Estudo qualitativo com base na busca dos confrontos e complementaridades presentes nos relatos dos sujeitos. A pesquisa foi realizada em duas Unidades de Atenção Primária à Saúde e dois Centros de Atenção Psicossocial. Os serviços de saúde mental, já há anos castigados pelo sub financiamento e desmonte, não têm sido capazes de fazer frente às crescentes demandas por atenção psiquiátrica. Pensar a cidadania envolve mudanças complexas nas instituições produtoras de cuidado em paralelo a mudanças também nas relações sociais, abrindo possibilidades importantes para a desconstrução do estigma criado em torno dos usuários de saúde mental. O acolhimento enquanto momento inicial de encontro entre o serviço é aquele que busca pelo cuidado representa um momento de pactuação e definição de fluxos, colocando em xeque a capacidade do serviço de dispor de seus recursos. O excesso de demandas e a forma como ela tem sido acolhida tem impactado na capacidade de reflexão e reorganização dos processos de trabalho, levando a um procedimento contínuo de reprodução do contexto.
\end{abstract}

Palavras-chave: Acolhimento; Saúde mental; Cuidado; Rede de atenção.

\begin{abstract}
The production of care in the field of mental health flourishes from the encounter between professionals and users. The study aims to analyze mental health care in the psychosocial care network from the practice of welcoming. Qualitative study based on the search for confrontations and complementarities present in the subjects' reports. The research was carried out in two Primary Health Care Units and two Psychosocial Care Centers. Mental health services, already punished by underfunding and dismantling for years, have not been able to meet the growing demands for psychiatric care. Thinking about citizenship involves complex changes in care-producing institutions in parallel with changes in social relationships as well, opening up important possibilities for deconstructing the stigma created around mental health users. Welcoming as the initial moment of meeting between the service is the one that seeks care represents a moment of agreement and definition of flows, putting into question the service's capacity to dispose of its resources. The excess of demands and the way in which it has been accepted has impacted on the capacity for reflection and reorganization of work processes, leading to a continuous procedure for reproducing the context.
\end{abstract}

Keywords: Reception; Mental health; Caution; Attention network.

\section{Resumen}

La producción de cuidados en el campo de la salud mental surge del encuentro entre profesionales y usuarios. El estudio tiene como objetivo analizar la atención de la salud mental en la red de atención psicosocial a partir de la práctica de la acogida. Estudio cualitativo basado en la búsqueda de enfrentamientos y complementariedades presentes en los informes de los sujetos. La investigación se llevó a cabo en dos Unidades de Atención Primaria de Salud y dos Centros de Atención Psicosocial. Los servicios de salud mental, castigados ya por la falta de financiación y el desmantelamiento 
durante años, no han podido satisfacer las crecientes demandas de atención psiquiátrica. Pensar en la ciudadanía implica cambios complejos en las instituciones productoras de cuidados en paralelo con cambios en las relaciones sociales, lo que abre posibilidades importantes para deconstruir el estigma creado en torno a los usuarios de salud mental. Acoger como momento inicial de encuentro entre el servicio es el que busca el cuidado representa un momento de concertación y definición de flujos, poniendo en duda la capacidad del servicio para disponer de sus recursos. El exceso de exigencias y la forma en que ha sido aceptado ha impactado en la capacidad de reflexión y reorganización de los procesos de trabajo, dando lugar a un procedimiento continuo de reproducción del contexto.

Palabras clave: Recepción; Salud mental; Precaución; Red de atención.

\section{Introdução}

A oferta do cuidado na seara da saúde mental floresce a partir do encontro entre usuários e profissionais. Esses momentos representam um solo fértil para a troca de experiências e consolidação de vínculos entre os sujeitos envolvidos, convergindo para uma produção intersubjetiva que intervém nas percepções de cuidado e colabora para produzir novos modos de agir em saúde (Franco; Merhy, 2012).

O acolhimento, termo por vezes utilizado para anunciar as relações entre profissionais e usuários, transcende esse conceito limitado por uma mera oferta de serviço. O ato de acolher em seu sentido mais amplo representa uma prática inerente à atuação profissional nos serviços de saúde, ampliando s possibilidades de atuação e desviando o foco de atenção da doença (Silva, et al, 2020).

Como a própria etimologia da palavra sugere, o acolhimento implica em receber e aceitar aquele indivíduo acolhido e suas demandas. Ao dar ouvidos a esse outro e admitir suas demandas como legítimas, o profissional abre espaço para novas e variadas análises do seu processo de trabalho, mobilizando-o no sentido de nortear fluxos, abrir as portas dos serviços e reconhecer o usuário como sujeito como agente ativo do seu processo de produção de saúde (Camargo; Castanheira, 2020).

Esta prática, cuja finalidade primordial é direcionar e dar as respostas adequadas as demandas que surgem ao longo da trajetória percorrida na busca do cuidado, envolve também habilidades que contemplem os aspectos clínicos, bem como a compreensão do funcionamento dos serviços e articulação dos dispositivos de cuidado, promovendo assim uma avaliação capaz de contemplar aspectos referentes ao sofrimento e as fragilidades do sujeito, de modo a produzir as ações voltadas para o cuidado (Coutinho; Barbieri; Santos, 2015; Rodrigues, 2018).

O ato de acolher representa, portanto, um aspecto indispensável para a qualificação do cuidado e operacionalização do acesso em suas diversas dimensões. O Sistema Único de Saúde (SUS) tem sua conjuntura atualmente constituída a partir de Redes de Atenção à Saúde que representam arranjos organizativos de ações e serviços de saúde de suma importância para avigorar a trajetória experimentada a partir do acolhimento enquanto diretriz operacional dos serviços de saúde, sendo esta ferramenta um aspecto imprescindível para ordenar sua demanda de acordo com as necessidades e prioridades e, não apenas, através da ordem de chegada ao serviço. Diante disso, o presente estudo tem por objetivo analisar o cuidado em saúde mental na rede de atenção psicossocial a partir da prática do acolhimento.

\section{Metodologia}

O estudo faz parte da pesquisa de doutorado intitulada: Articulação do cuidado na rede de atenção psicossocial: interface com as políticas de saúde mental. É uma investigação qualitativa cuja finalidade foi identificar as discordâncias e reciprocidades presentes nas falas dos sujeitos, de acordo com os preceitos da hermenêutica crítica (Minayo, 2013).

Os cenários do estudo consistiram em duas Unidades de Atenção Primária à Saúde (UAPS) e dois Centros de Atenção Psicossocial das Secretarias Executivas Regionais II e VI do município de Fortaleza, capital do Ceará. A escolha por essas duas regionais se deu em virtude do acordo consolidado junto ao Sistema Municipal de Saúde Escolar. Ademais, estes territórios conservam entre si diferenças marcantes no que concerne ao Índice de Desenvolvimento Humano (IDH). O estreitamento de 
laços com o serviço, entrada no campo e coleta de dados ocorreu no período de novembro de 2020 a janeiro de 2021. Integraram o estudo dezesseis sujeitos, sendo quatro usuários; quatro trabalhadores da APS, compondo equipes de Estratégia Saúde da Família, a saber: dois médicos e dois enfermeiros. No CAPS foram entrevistados 8 profissionais, sendo 1 médico, 3 enfermeiras, 2 psicólogas e 2 assistentes sociais.

Os critérios elencados para a escolha dos trabalhadores de saúde participantes da pesquisa foram: exercer o cargo atual pelo período mínimo de um ano. Essa linha demarcatória de tempo foi estimada por acreditar-se que permite um maior envolvimento e compreensão desses atores acerca de seus locais de trabalho. Para os usuários os critérios estabelecidos foram estar sendo sistematicamente acompanhado no serviço por, pelo menos, seis meses e estar fazendo o uso regular dos psicofármacos prescrito.

O número de participantes teve por base a relevância de suas falas e contribuições ao objeto de estudo, com base nos aspectos pertinentes à sua abordagem interpretativa. Foram excluídos da pesquisa os profissionais que estavam gozando de férias durante a coleta e os usuários cujo quadro clínico não lhes possibilitou responder aos questionamentos.

Os discursos foram apreendidos através da técnica de entrevista semiestruturada que priorizou questionamentos a respeito dos aspectos relacionados ao acolhimento em saúde mental, atividades desenvolvidas nos serviços com vistas à atenção psicossocial e os desafios enfrentados durante a oferta de cuidado em saúde mental. Já os usuários foram questionados acerca das ações de cuidado que os serviços disponibilizam, como se processavam as relações junto aos profissionais de saúde e que desafios são encontrados na trajetória na busca pelo cuidado em saúde mental.

As entrevistas foram integralmente gravadas em dispositivos de áudio mediante permissão dos entrevistados e assinatura do Termo de Consentimento Livre e Esclarecido (TCLE). Após isso foram transcritas e codificadas com as letras Med, para médico; Enf APS, para enfermeiro da atenção primária; Enf CAPS para os integrantes do centro de atenção psicossocial; Psi para psicólogo; AS para assistente social e U para usuário; com numeral arábico na sequência, seguindo a ordem de aplicação das entrevistas. A observação livre com registro em diário de campo também foi utilizada durante a pesquisa.

A análise do material empírico tomou por base a hermenêutica crítica, conforme proposta por Minayo (2013) e reinterpretada por Assis e Jorge (2010), lançando mão das etapas de ordenação, classificação e análise final dos dados.

Os resultados apresentados preservam a identidade dos participantes, sendo respeitados os preceitos éticos conforme indicado pela Resolução n ${ }^{\circ}$ 466, de 12 de dezembro de 2012 (2013). Esta pesquisa foi aprovada pelo Comitê de Ética em Pesquisa com Seres Humanos da Universidade Estadual do Ceará, por meio do Parecer nº 3.676.599.

\section{Resultados e Discussão}

Os cenários do estudo são serviços responsáveis por uma população adstrita de número expressivo, entretanto podemos destacar diferenças marcantes entre os locais pesquisados, a despeito do número de usuários presentes em sua área de abrangência. A secretaria executiva regional II comporta alguns dos bairros com o maior índice de desenvolvimento humano da capital cearense, em contrapartida, a VI vive um outro extremo dessa realidade ao comportar bairros com os menores índices de desenvolvimento e maiores condições de violência e vulnerabilidade social. Informações dessa natureza são relevantes pois repercutem sobre os usuários e consequentemente sobre suas demandas, afetando também a relação dos usuários com o serviço e seu padrão de uso.

\section{Lutas e acasos na busca por acolhimento}

O contexto pelo qual o Brasil passa, tem sido marcado pela recessão econômica, elevação do custo de vida, bem como a precarização das condições de trabalho, circunstâncias estas que tem impactado sobremaneira o estilo de vida no país, levando 
ao aprofundamento da pobreza e da desigualdade social. Essa já difícil conjuntura intensificou-se ainda mais a partir do ano de 2020 com a pandemia do novo coronavírus, aumentando a incidência de transtornos psíquicos na população (Lopes, 2020).

Atualmente, dados bastante consistentes já revelam uma associação entre certos níveis de pobreza e vulnerabilidade social com a ocorrência de transtornos mentais (Lopes, 2020). Diante deste fato não é de se gerar espanto que sujeitos que vivem nessas circunstâncias acabem por requerer mais cuidados aos serviços de atenção psicossocial, entretanto o que se pode observar é que a oferta dos serviços não se dilata na mesma proporção que a demanda.

Concernente a isso, é importante evidenciar que os serviços de saúde mental, já há anos castigados pelo sub financiamento e desmonte, não têm sido capazes de fazer frente às crescentes demandas por atenção psiquiátrica (Cruz, Gonçalves, Delgado, 2020). As Unidades de Atenção Primária, por sua vez, embora potentes para atuar frentes os transtornos leves, vem lidando com uma demanda bastante acrescida em virtude do cenário pandêmico. Somam-se a isso os antigos problemas relacionados à desarticulação entre os serviços e a resistência em responsabilizar-se pelas demandas de saúde mental (Carvalho, et al, 2017).

O estudo nos revelou que, a despeito de os serviços primários se esforcem no sentido de produzir cuidado em saúde mental, essa atenção é pontual e a oferta de cuidado tem seu enfoque reduzido na prescrição medicamentosa, realidade também compartilhada pelos centros de atenção psicossocial: "De um modo geral, eu vejo que a gente não consegue cumprir o que é a proposta da Reforma Psiquiátrica [...] Me questiono, inclusive, se não atuamos no nível do ambulatório, uma fábrica de receitas (AS1).

De encontro a isso, nas percepções enunciadas pelos profissionais entrevistados, o cuidado é sinalizando enquanto um conjunto de ações que envolvem escuta e desvelo e cujo cerne são as demandas dos usuários, aspectos que convergiram na maioria dos discursos dos profissionais, tanto da rede básica quanto da atenção psicossocial: "Cuidado pra mim significa se doar pelo outro, captar aquilo que ele traz, o que causa sofrimento e batalhar no sentido de ajudar" (Enf APS 2). "Cuidado em Saúde Mental eu entendo que seja compreender o usuário do sistema acima de qualquer coisa como um cidadão de direitos, cidadão contribuinte, que justifica a existência da política, e que deve ser visto em todos os aspectos possíveis" (Psi 1).

Observamos emergir aqui um aspecto importante no que diz respeito à cidadania dos usuários de saúde mental, desconstruindo a noção clássica limitada de uma cidadania que prega uma inclusão social forjada através da igualdade formal e jurídica dos sujeitos. Segundo Silva e Rosa (2019), uma cidadania ampliada implica pensar em um conceito que transcenda a noção de tratar a todos de forma igual, abrindo espaços dialógicos que permitam a incorporação e o respeito às diferenças.

Pensar essa cidadania envolve mudanças complexas nas instituições produtoras de cuidado em paralelo a mudanças também nas relações sociais, abrindo possibilidades importantes para desfazer o estigma criado em torno dos usuários de saúde mental.

Um aspecto que chama a atenção nos discursos para além da noção de cuidado enquanto ações que compreendem a reponsabilidade pelos usuários de seu território e a necessidade de otimizar seus recursos, é o fato de o usuário ser apontado em algumas circunstâncias como indivíduo destituído de saber acerca de suas demandas, requerendo uma espécie de tutela por parte do profissional de saúde.

"O trabalho em saúde é muito complexo porque o usuário é que vai dizer o que ele precisa. O grande problema é que na maioria das vezes ele não sabe de fato o que ele precisa. Ele chega aqui e quer a receita, mas a gente sabe que só o remédio não é tudo [...] Por outro lado é até mais fácil porque não temos vários outros recursos" (Enf. APS1).

Esse discurso sinaliza a presença da assimetria ainda fortemente cultivada nas relações entre profissionais e usuários. Os profissionais exercem o poder que lhes foi conferido através de um diploma e que é, no dia a dia, legitimado pela relação de subordinação fomentada com aqueles que buscam assistência nos serviços, deixando de lado aspectos importantes como o 
intermédio da informação nesse processo de interação. Observamos neste ensejo a produção de barreiras no intercâmbio entre profissional e usuário, privando este do direito básico de requerer informações a respeito de seu estado de saúde e acerca do cuidado que lhe é oferecido (Caminha, et al, 2021).

Ademais, soma-se a isso limitações na atenção recebida tanto pela dificuldade de acessar o serviço quanto pelo fato de longitudinalidade do cuidado não ser assegurada: “Conseguir já é um milagre, mas às vezes tem algum problema com o remédio, a gente tem que enfrentar tudo de novo pra conseguir" (U. 3).

Percebemos aqui a ausência de fluxos que regulem o acesso aos serviços, dificultando o transitar dos usuários dentro deles e mais ainda dentro da rede. Os próprios profissionais salientam que a ausência de fluxos demarcados resulta em um menor potencial de uso dos serviços:

Então antes de olhar mesmo até para fora, vejo que a gente precisava trabalhar até mesmo dentro para gente ficar único com o mesmo discurso e assim se fortalecer para o momento de levar a nossa pauta para os outros serviços. Cada uma hora a gente fala uma coisa ou cada serviço fala uma coisa (Psi 2).

Entretanto, mesmo ante os diversos obstáculos enfrentados para lograr êxito na busca pelo cuidado, quando conseguem ser acolhidos, esses usuários atribuem qualidade ao serviço e tem suas expectativas de cuidado correspondidas: "Tô aqui não sei nem desde que horas, mas consegui ser acolhido e vou conseguir minha receita, então estou satisfeito. Infelizmente, isso é SUS" (U1). "Quando consigo ser atendida me sinto cuidada (U4).

Sabe-se que a qualidade é um conceito próprio de cada indivíduo, sendo influenciado por suas percepções e subjetividades e muito embora o SUS represente um direito social assegurado pelo Estado, permanece na concepção de muitos, especialmente das populações mais vulneráveis, a ideia de que a atenção prestada tem ares de caridade, sendo o fato de conseguir ser atendido e ter sua receita renovada o padrão ouro de referência para considerar o serviço bom ou não (Gaino, et al, 2018).

Outro aspecto importante que foi identificado diz respeito a forma como os serviços têm se organizado para acolher os que buscam atendimento, especialmente os Centros de Atenção Psicossocial presentes em áreas mais vulneráveis, fato que tem sido um capítulo à parte, na medida em que, em alguns estabelecimentos, a recepção atua como uma predecessora do processo de escuta, definindo quem será acolhido e ferindo a proposição de que todo usuário tem direito à escuta. Esta definição, em um primeiro momento, toma por critério a ordem de chegada dos usuários ao serviço, uma vez que existe um número predeterminado de vagas disponíveis para o acolhimento a cada dia. Dessa forma, aqueles que chegam mais cedo tem assegurado o seu direito de ser acolhido por um profissional: "Temos uma quantidade enorme de pacientes aqui diariamente, impossível acolher todos" (Enf CAPS 3); "Conforme a ordem de chegada, a recepção distribui as fichas" (Psi).

$\mathrm{O}$ acolhimento, em tese, seria uma ferramenta usada para suplantar a ideia da ordem de chegada, entretanto a própria definição pela recepção de quem terá o direito de ser acolhido reafirma essa lógica. O momento de encontro inicial entre usuário e serviço é marcado por tensionamentos, uma vez que os recepcionistas estabelecem quem será acolhido, sendo o critério elencado para tanto, a ordem de chegada ao serviço, o excedente é então dispensado e aconselhado a retornar no dia seguinte.

A pesquisa nos revelou ainda que a forma como o excedente da demanda é direcionada varia conforme as características do próprio território, sendo marcada por diferenças expressivas nos diferentes serviços estudados. Em áreas com menor vulnerabilidade e maior índice de desenvolvimento, observou-se a adoção da estratégia de um "profissional de porta". Esse profissional fica responsável por realizar a escuta daqueles pacientes que não conseguiram ser acolhidos, identificando, portanto, se aquela demanda pode esperar ou se apresenta um caráter urgente, como em circunstâncias de ideação suicida.

Já os dispositivos de saúde das áreas periféricas remontam a uma realidade bem distinta, com cenários marcados pela escassez de profissionais, demanda excessiva de sujeitos submetidos às condições de vulnerabilidade expressivas e esgotamento das equipes de atenção, descortinando a realidade de serviços que lutam para sobreviver. 
Neste panorama de desaparelhamento em que vivem os serviços presentes nas comunidades mais pobres, constatou-se que a demanda que excede sua programação diária acaba não sendo oportunamente gerenciada, ficando parte considerável dos usuários sem a devida assistência. Tem-se, portanto, ferida a dimensão do acesso denominada "disponibilidade", caracterizada nesse contexto como a disposição de um determinado serviço ao alcance dos usuários (Assis; Jesus, 2012).

Este contexto nos revela ainda que os usuários têm limitadas as suas possibilidades de acessar o serviço e serem acolhidos em virtude de características como disponibilidade de profissionais para atendimentos, bem como a capacidade do serviço de acolher demandas que não estavam previamente programadas.

Corroborando os achados de Assis e Jesus (2012), ao considerarmos que a busca por atendimento se divide em fases e superar as barreiras impostas pela demanda excessiva e baixa disponibilidade de profissionais representa a primeira fase a ser vencida pelos usuários na busca por assistência. Entretanto, essa é apenas a primeira barreira a ser vencida, conforme destacado no seguinte discurso: "Cheguei aqui antes de seis [...] tem que falar com algum deles pra marcar a consulta ou pra renovar receita" (U.2).

Nos cenários de estudo foi possível identificar duas interfaces distintas do acolhimento. Em serviços responsáveis por uma população em maior vulnerabilidade observou-se uma procura por atenção que extrapola a capacidade instalada do serviço, sendo o encaminhamento utilizado como uma ferramenta a desresponsabilização. Em cenários cuja demanda e oferta apresentavam maior proporcionalidade foi possível observar o acolhimento enquanto ferramenta que, como o próprio termo sugere, recebe, abraça o paciente e suas demandas: "Nesse momento do acolhimento buscamos mesmo colocar à disposição do paciente todo o arsenal que o serviço tem disponível pra encontrar o que melhor se adequa ao interesse dele" (Psi 2); "Temos um fluxo muito grande, dificuldade com médico e a solução é encaminhar para à saúde da família, para o programa de saúde mental" (Enf CAPS 1).

Diante disso, é perceptível que apesar de superar a barreira inicial e conseguir acessar o serviço e seus profissionais, não se pode presumir que o cuidado conseguiu ser de fato acessado pelo usuário. Isso acontece, pois, ao cultivar relações verticalizadas fortalecidas a partir do poder/saber profissional, ao dispor do uso excessivo de psicotrópicos e fundamentar suas ações essencialmente no encaminhamento a outros serviços, o usuário se vê despido de sua singularidade e suas necessidades não são mais o ponto que norteia o cuidado, sendo este conduzido conforme as demandas do serviço de saúde.

\section{Da utopia do acolher à realidade do produtivismo}

$\mathrm{O}$ acolhimento enquanto momento inicial de encontro entre o serviço e aquele que busca pelo cuidado representa um momento de pactuação e definição de fluxos, colocando em xeque a capacidade do serviço de dispor de seus recursos. Acerca disso, os entrevistados sinalizaram a importância de assumir uma postura ética e acolhedora aos usuários, entretanto salientaram o desgaste implicado no processo: "Tentamos receber esse paciente da forma mais gentil possível, por que sabemos que ela já chega com toda uma carga" (AS 2); "Não é tão simples. Em alguns momentos a gente precisa mobilizar vários elementos da rede pra tentar resolver o problema de um usuário. Leva um tempo que nem sempre temos e o esgotamento às vezes é imenso" (Psi 1).

O processo de escuta e gerenciamento das demandas perpassa a formação de relações entre usuários e profissionais e esse momento de encontro impele o profissional a lançar mão de seu conhecimento e capacidade dialógica para ofertar a resposta requerida pelo usuário naquele momento (Silva, 2020).

Ainda sobre isso, profissionais salientaram que, por diversas vezes, a resolução das demandas trazidas ao serviço extrapola sua capacidade de atuação, tropeçando em barreiras tais como agendas médicas lotadas e disponibilidade de ações terapêuticas não centradas no uso medicamentoso: "É desgastante. Algumas vezes o usuário não está se dando bem com a medicação, eles chegam desesperados e só tem vaga na agenda médica pra dois meses depois, a opção é encaminhar" (Psi 1); "É 
uma quantidade muito grande de pacientes para atender, tem um aqui dentro e vários lá fora esperando. Tem dias que passo a manhã aqui e não tomo nem água" (Enf CAPS 2).

Sobre isso, sabe-se que o excesso de estímulos e a velocidade das informações leva os indivíduos à realização simultânea de múltiplas tarefas em busca do "desempenho máximo", e acaba por prejudicar o que Han (2015) denominou de aprofundamento contemplativo. A demanda acentuada e o número expressivo de pacientes têm saturado profissionais, levando-os a buscar soluções mais simplistas como medida estanque para amortecer a sobrecarga no trabalho.

$\mathrm{O}$ alto número de pacientes que procuram o serviço faz com que este busque adequar seus processos produtivos de modo a assistir o maior número possível de sujeitos. Ademais, os serviços de um modo geral têm trabalhado na perspectiva do alcance de metas previamente estipuladas, atendendo de tal forma aos interesses da típica lógica do capitalismo.

Tal contexto deflagra a fragilidade das políticas públicas bem como da gestão dos serviços, impondo aos profissionais um cenário de trabalho mecanicista e racionado. A influência do capitalismo nos processos produtivos aguça o alcance de metas, que para serem atingidas demandam a rotinização e a segmentação dos processos de trabalho em saúde, conduzindo os profissionais ao produtivismo enquanto lógica de mercado (Teston, 2018).

Esse fenômeno, fundamentado com base em um raciocínio forjado a partir do competitivismo típico do capitalismo, confere destaque e importância a aspectos de ordem quantitativa relacionados ao resultado e desempenho. Essas questões vão de encontro ao tempo e às condições requeridas para que novas ações e novos saberes sejam produzidos, subvertendo a lógica produtivista em prol da atenção e desvelo demandado pelo cuidado em saúde mental.

Esse mecanismo de trabalho imposto de pelo produtivismo em saúde, acaba limitando o tempo de atendimento dedicado a cada paciente, empobrecendo a qualidade da assistência prestada. Cenários como o estudado revelam um contexto que sobrepuja os usuários, sua história, suas características e singularidade.

$\mathrm{O}$ acolhimento enquanto atendimento pontual revelou-se falho no seu intento de abraçar as demandas e a partir do conhecimento das necessidades e condições de vida dos sujeitos, nortear os fluxos dos usuários dentro da rede e ampliar suas possibilidades de êxito na busca pelo cuidado, limitando suas ações ao encaminhamento indiscriminado a outros dispositivos: "A demanda é muito grande e as vezes é um caso que você vê que teria um resultado melhor com outras alternativas, um grupo, uma terapia, mas não tem suporte" (Enf APS 2).

Os discursos dos profissionais descortinam uma série de fragilidades dos serviços e da própria lógica de atuação que os regem para ofertar uma outra possibilidade terapêutica cujo cerne não esteja centrado na prescrição medicamentosa. A falta de alternativas terapêuticas que possam adjuvar no tratamento tem frustrado profissionais, levando-os a crer que, muitas vezes, o seu processo de trabalho implica tão somente em "enxugar gelo" (Med.1), expressão popularmente proferida para referir-se a algo que não produz quaisquer resultados.

Estamos diante, portanto, de uma circunstância de contato pouco frutífera no sentido da produção do bom encontro aquele que surge mediante o incremento da potência de ação dos envolvidos, nesse caso, profissionais e usuários. Sobre isso, é válido destacar que, muito embora não seja possível e até mesmo viável fugir completamente do fluxo demanda-oferta, é necessário buscar estratégias inventivas capazes de ressignificar os processos de trabalho enquanto possibilidades esperando para serem descobertas.

Para isso, portanto não podemos esquecer do papel da gestão em prover condições que permitam um melhor gerenciamento do trabalho e dos fluxos de assistência que desconexos, obstruem o trânsito dos usuários nos equipamentos, reprimindo a demanda e produzindo lacunas assistenciais importantes: "A gente chega aqui antes das seis pra conseguir a vaga nesse acolhimento e eles mandam a gente para o posto com encaminhamento, as vezes eles atendem, as vezes mandam a gente de volta" (U 3). 
O contexto da pandemia ampliou essas lacunas assistenciais e expôs de forma ainda mais clara o desmantelamento dos fluxos entre os serviços. Atualmente as unidades de atenção primária do município tem voltado seus esforços prioritariamente à atenção de pacientes com casos suspeitos de COVID. Entretanto este fato tem colaborado para produzir uma demanda reprimida na agenda de saúde mental da UAPS, levando esses indivíduos, na esperança de ter sua necessidade sanada, a buscar os CAPS como alternativa: “Agora com o COVID estamos meio quem sem norte. As agendas aqui estão lotadas e no posto eles só estão atendendo COVID. O que a gente faz? Pra onde mandar?" (Psi 1); "Na primeira onda da pandemia as receitas especiais passaram a valer seis meses e as receitas azuis, dois. Ajudava a gente a desafogar o sistema. Agora não, todas as receitas só valem um mês" (Enf APS 2). "Tem usuário que chega aqui pra pegar remédio e descobre na hora que a validade das receitas mudou, por que é assim, a gestão resolve da noite para o dia e ficamos sem ter o que fazer" (Psi 2).

Esse cenário nos chama atenção para as repercussões da falta de fluxos claros entre os serviços, bem como um acolhimento ineficiente enquanto uma estrutura responsável por engendrar o fenômeno da sobremedicalização (Freitas, 2017; Tesser, 2019).

As consultas cronometradas, o desgaste profissional e os recursos terapêuticos escassos conferem à assistência uma espécie de automatismo que vai de encontro à lógica de uma atenção acolhedora e resolutiva. Essa discussão nos revela algo bastante comum no cenário brasileiro, o fato de que a mera existência de um instrumento legal não garante por si só sua implementação na prática.

Muito embora tenhamos todo um arsenal jurídico que regulamente a rede de atenção psicossocial e que determine o direito do usuário a uma assistência universal e equitativa, o que observamos na prática são mecanismos que mediam o acesso de modo seletivo e, por vezes, excludente, limitando o acesso dos usuários aos serviços de saúde. Ademais, lacunas assistenciais importantes são produzidas em virtude da desarticulação entre dispositivos da rede de atenção psicossocial, mais expressivamente entre CAPS e APS, repercutindo na forma de operar o cuidado nesses territórios.

\section{Considerações Finais}

Os achados da pesquisa revelam que a desproporção entre demanda por cuidados e disponibilidade de recursos no serviço vem culminando em cenários de sobrecarga e esgotamento profissional, culminando na contínua desresponsabilização pelos usuários assistidos, bem como produzindo extensos vazios assistenciais no contexto da atenção psicossocial. O excesso de demandas e a forma como ela tem sido acolhida tem impactado na capacidade de reflexão e reorganização dos processos de trabalho, levando a um procedimento contínuo de reprodução do contexto.

É válido ressaltar que o estudo se limitou a tecer análises acerca do cuidado e o acolhimento em saúde mental em apenas quatro dispositivos da rede de atenção psicossocial, sendo necessária a realização de estudos mais extensos na RAPS com vistas a compreender de forma mais ampla de que maneira o cuidado se processa e que fatores influenciam nisso.

Daremos continuidade a pesquisa relacionada a temática do cuidado na saúde mental, fortalecendo o conhecimento.

\section{Referencias}

Assis, M. A. A., \& Jorge, M. S. B. (2010). Métodos de análise em pesquisa qualitativa. In: Santana, J. S. S. S \& Nascimento, M. A. A, organizadores. Pesquisa: métodos e técnicas de conhecimento da realidade social. Ed. UEFS, 139-59.

Assis, M. A. A., \& Jesus, W. L. A. (2012). Acesso aos serviços de saúde: abordagens, conceitos, políticas e modelo de análise. Ciênc. Saúde Coletiva, v.17 (11).

Camargo, S. D., \& Castanheira, E. R. L. (2020). Ampliando o acesso: o Acolhimento por Equipe como estratégia de gestão da demanda na Atenção Primária à Saúde (APS). Interface, 24 (1).

Caminha, E. C. C. R., et al (2021). Relações de poder entre profissionais e usuários da Atenção Primária à Saúde: implicações para o cuidado em saúde mental. Saúde Debate, v.45 (128).

Carvalho, M. F. A. A., et al (2017). Desarticulação da rede psicossocial comprometendo a integralidade do cuidado. Rev. Esc. Enferm. USP 51. 
Research, Society and Development, v. 10, n. 10, e399101019046, 2021

(CC BY 4.0) | ISSN 2525-3409 | DOI: http://dx.doi.org/10.33448/rsd-v10i10.19046

Conselho Nacional de Saúde. Resolução nº 466, de 12 de dezembro de 2012 (2013). Dispõe sobre diretrizes e normas regulamentadoras de pesquisas envolvendo seres humanos. Diário Oficial da União. 13 Jun 2013.

Coutinho, L. R. P., Barbieri, A. R., \& Santos, M. L. M. (2015). Acolhimento na atenção primária à saúde: revisão integrativa. Saúde Debate, 39(105), 514-24.

Cruz, N. F. O., Gonçalves, R. W., \& Delgado, P. G. G. (2020). Retrocesso da reforma psiquiátrica: o desmonte da política nacional de saúde mental brasileira de 2016 a 2019. Trab. Educ. Saúde, v.18 (3)

Franco, T. B. F., \& Merhy, E. E, (2012). Cartografias do Trabalho e Cuidado em Saúde. Tempus Actas de Saúde Coletiva, v. 6(2).

Freitas F., \& Amarante P. (2017). Medicalização em psiquiatria. 2. ed. Rio de Janeiro: Editora Fiocruz.

Gaino, L. V, et al (2018). O conceito de saúde mental para profissionais de saúde: um estudo transversal e qualitativo*. SMAD, Rev. Eletrônica Saúde Mental Álcool Drog. (Ed. port.), Ribeirão Preto, v. 14(2),108-116.

Han, B-C. (2015). Sociedade do cansaço. Tradução de Enio Paulo Giachini. Vozes.

Lopes, C. S (2020). Como está a saúde mental dos brasileiros? A importância das coortes de nascimento para melhor compreensão do problema. Cad. Saúde Pública, 36 (2).

Minayo, M. C. S. (2013). O desafio do conhecimento: pesquisa qualitativa em saúde. (13a ed.), Hucitec.

Rodrigues, J. B. (2018). O acolhimento na atenção básica: desafios e avanços no município de São Bernardo do Campo [mestrado]. São Paulo: Instituto de Saúde.

Silva, S. L. C., \& Rosa, L. C. S. (2019). Cidadania da pessoa com transtorno mental: avanços e impasses na dinâmica da reforma psiquiátrica brasileira. Humanidades e Inovação. v. 6(17).

Silva, T. C. F. et al (2020). Acolhimento noturno em um Centro de Atenção Psicossocial III. Rev. Bras. Enferm.,73 (1).

Tesser, C. D. (2019). Cuidado clínico e sobre medicalização na atenção primária à saúde. Trab. Ed. Saúde [internet].

Teston, L. M., et al (2018). Avaliação no SUS: uma crítica à ideologia do produtivismo no capitalismo contemporâneo. Saúde debate, 42 (sup3). 